\title{
Local mapping of dissipative vortex motion
}

\author{
B. Raes, ${ }^{1}$ J. Van de Vondel, ${ }^{1}$ A. V. Silhanek, ${ }^{2}$ C. C. de Souza Silva, ${ }^{3}$ J. Gutierrez, ${ }^{1}$ R. B. G. Kramer, ${ }^{4}$ and V. V. Moshchalkov ${ }^{1}$ \\ ${ }^{1}$ INPAC-Institute for Nanoscale Physics and Chemistry, Nanoscale Superconductivity and Magnetism Group, \\ KU Leuven, Celestijnenlaan 200D, B-3001 Leuven, Belgium \\ ${ }^{2}$ Département de Physique, Université de Liège, Allée du 6 août 17, B5, B-4000 Sart Tilman, Belgium \\ ${ }^{3}$ Departamento de Fisica, Universidade Federal de Pernambuco, Cidade Universitaria, 50670-901 Recife-PE, Brazil \\ ${ }^{4}$ Institut Néel, CNRS, Université Joseph Fourier, BP 166, 38042 Grenoble Cedex 9, France
}

(Received 24 May 2012; published 23 August 2012)

\begin{abstract}
We explore, with unprecedented single vortex resolution, the dissipation and motion of vortices in a superconducting ribbon under the influence of an external alternating magnetic field. This is achieved by combining the phase sensitive character of ac susceptibility, allowing us to distinguish between the inductive and dissipative responses, with the local power of scanning Hall probe microscopy. Whereas the induced reversible screening currents contribute only inductively, the vortices do leave a fingerprint in the out-of-phase component. The observed large phase-lag demonstrates the dissipation of vortices at time scales comparable to the period of the driving force (i.e., $13 \mathrm{~ms}$ ). These results indicate the presence of slow microscopic loss mechanisms mediated by thermally activated hopping transport of vortices between metastable states.
\end{abstract}

DOI: 10.1103/PhysRevB.86.064522

PACS number(s): 74.78.-w, 74.25.F-, 74.25.Wx, 74.40.Gh

The universal problem of energy dissipation embodies the irreversible conversion of work into heat in a dynamic system. This is fundamentally connected with the inequality present in the second law of thermodynamics, expressing a monotonic increase of the entropy or the fact that a perpetual motion machine of the second kind does not exist. ${ }^{1}$ The macroscopic quantum effect of superconductivity is the closest thing to the idiom of a perpetual motion machine. Indeed, due to the presence of an energy gap between the sea of condensed Cooper pairs and the fermionic quasiparticles, electrical current can circulate forever in a superconducting ring. ${ }^{2}$ However, when a type II superconductor is in the mixed state, the motion of vortices, perpendicular to supercurrents, results in a resistive voltage drop and the unique hallmark of dissipationless transport collapses. ${ }^{3}$

In general, whenever a dissipative system is subjected to a periodic excitation, e.g., a crystal to electromagnetic radiation or a driven damped harmonic oscillator, the periodic force will perform work to drive the system through subsequent dissipative cycles. The dissipative or frictional component of the system, related to a nonconservative force, will induce a phase shift between the response and the external drive. For example, the imaginary part of the relative permittivity is closely related to the absorption coefficient of a material ${ }^{4}$ or a phase-lag appears in the motion of a damped harmonic oscillator. This close connection between dissipation of energy and the out-of-phase component of the system's response is used in spectroscopic measurements to gain information concerning the nature and efficiency of the dissipation processes and is in a one-to-one relationship with the system's equilibrium fluctuations through the fluctuation dissipation theorem. ${ }^{5}$

To investigate the dissipative nature of electrical transport, when a superconductor is in the mixed state, the integrated response of the superconductor under an external alternating magnetic field is recorded in measurements of the global ac susceptibility. ${ }^{6}$ The action of the alternating magnetic field will result in a complex response arising from a collection of two contributors: the screening currents and the vortices.
In the first approximation one can study only the linear response, which is completely determined by a measurement of the real and imaginary part of the complex relative permeability. These Fourier components are mutually connected by the Kramers-Krönig relationships. The real part describes the in-phase response of the magnetic induction to the external magnetic ac field and is related to the macroscopic shielding abilities. The imaginary part describes the out-of-phase response of the magnetic induction, arising as indicated before, necessarily from dissipative ac losses within the superconductor. The dependencies of these two response functions on changing thermodynamical variables, such as temperature and dc external magnetic field, or the ac-excitation parameters, such as the amplitude and the frequency, provide very valuable information concerning the pinning efficiency and reveal the fingerprints of the particular ac dynamic phases the vortex lattice exhibits. ${ }^{7-9}$ Since the recorded signal represents an average over all present flux lines and screening currents in the sample, the link with the microscopic ac reponse is indirect. Pioneering theoretical works ${ }^{10-12}$ have contributed substantially to link this global response to the microscopic vortex dynamics and/or the ac-field penetration.

However, until now the dissipation of individual vortices remains concealed, despite the enormous amount of theoretical research. In this work we combine the strength of phase sensitive detection used in global ac-susceptibility measurements ${ }^{6}$ and the power of individual vortex visualization, accessible with scanning Hall probe microscopy (SHPM),${ }^{13-16}$ to reveal the microscopic linear response of a type II superconductor to an external applied ac magnetic field. The local character of this scanning ac-susceptibility microscopy (SSM) technique and the argument "seeing is believing" allow us to bridge the gap between the global ac-susceptibility measurements and their associated microscopic theories of vortex motion and ac-field penetration and the real microscopic ac response.

In SSM, we continuously excite the sample with an external ac magnetic field, $h_{\mathrm{ac}}(t)=h_{\mathrm{ac}} \cos (\omega t)$. The Hall voltage, $V_{H}(x, y, t)$, measured locally by a Hall microprobe, is picked 
up by a lock-in amplifier. The excitation signal for the external applied ac field, feeds a phase-locked loop which extracts the in-phase, $V_{1}^{\prime}(x, y)$, and quadrature components, $V_{1}^{\prime \prime}(x, y)$, of $V_{H}(x, y, t)$. In the first approximation these are, respectively, proportional to the in-phase, $b_{\mathrm{ac}}^{\prime}(x, y)$ and out-of-phase, $b_{\mathrm{ac}}^{\prime \prime}(x, y)$, ac components of the local magnetic induction, $b_{z}(x, y, t)$, coarse grained by the size of the cross. We can introduce two response parameters which completely determine the local linear response of $b_{z}(x, y, t)$ to $h_{\text {ac }}(t)$. We define the real and imaginary part of the local relative permeability, $\mu_{r}(x, y)$, as ${ }^{17}$ :

$$
\begin{aligned}
\mu_{r}(x, y) & =\mu_{r}^{\prime}(x, y)-i \mu_{r}^{\prime \prime}(x, y) \\
& =\frac{1}{h_{\mathrm{ac}}}\left[b_{\mathrm{ac}}^{\prime}(x, y)-i b_{\mathrm{ac}}^{\prime \prime}(x, y)\right] .
\end{aligned}
$$

Just as in the global ac-susceptibility technique, the in-phase component, $\mu_{r}^{\prime}(x, y)$, is related to the local inductive response, while the out-of-phase component, $\mu_{r}^{\prime \prime}(x, y)$, is related to microscopic ac losses. SSM provides a spatial map of these two Fourier components and describes, as such, the local linear response of $b_{z}(x, y, t)$ to $h_{\mathrm{ac}}(t)$ with single vortex resolution.

The mapping of $b_{z}(x, y)$ was obtained using a modified lowtemperature SHPM from Nanomagnetics Instruments. The typical scan area at $4.2 \mathrm{~K}$ is $16 \times 16 \mu \mathrm{m}^{2}$. The collinear dc and ac external magnetic fields are always applied perpendicular to the sample surface. The investigated sample is a $\mathrm{Pb}$ ribbon of $0.5 \mathrm{~mm}$ long, $50 \mathrm{~nm}$ thick, and $9 \mu \mathrm{m}$ wide and exhibits a superconducting transition at $T_{c}=7.20 \mathrm{~K}$. As the signal picked up by the Hall probe contains different contributions, arising from the screening currents, the vortex signals and the external field itself, the measured local linear ac response is also determined by all contributing factors. This particular sample design allows us to map the spatial dependence of the linear response to $h_{\mathrm{ac}}(t)$, covering the whole width of the sample in a single scanning area, including the Meissner response at the sample border and the vortex motion deeper into the ribbon volume.

Figure 1(a) shows a SHPM image of a vortex distribution obtained after performing a field cool (FC) in $H=0.13 \mathrm{mT}$ to $T=6.7 \mathrm{~K}$ while an external field with $h_{\mathrm{ac}}=0.1 \mathrm{mT}$ and $f=77.123 \mathrm{~Hz}$ is continuously applied. The resulting vortex distribution, obtained by performing a FC experiment, corresponds to a frozen vortex structure nucleated close to $T_{c}$. ${ }^{18}$ The $\mathrm{FC}$ process forces vortices to nucleate at the strongest pinning sites and results in a nonsymmetrical vortex distribution. The external ac field shows up as an additional monochromatic noise in the SHPM images getting more pronounced for temperatures close to $T_{c}$. However, for all investigated temperatures the average vortex positions do not change, indicating that for $h_{\mathrm{ac}}=0.1 \mathrm{mT}$ the resulting average vortex response is limited to displacements below the experimental spatial resolution.

Figure 1(b) shows a representative set of simultaneously acquired SSM images of $\mu_{r}^{\prime}(x, y)$ (top row) and $\mu_{r}^{\prime \prime}(x, y)$ (bottom row), respectively describing the inductive and dissipative response, when the temperature is decreased progressively from $T=7 \mathrm{~K}$ to $T=6.7 \mathrm{~K}$. A first straightforward observation is that at the edges of the scan area, meaning relatively far away from the $\mathrm{Pb}$ ribbon, the local induction oscillates perfectly in-phase with $h_{\mathrm{ac}}(t)$. A clear paramagnetic response, $\mu_{r}^{\prime}(x, y)>1$, is visible at the edge of the $\mathrm{Pb}$ ribbon, where the response is dominated by the induced screening currents. This enhancement of the external ac field is caused by a strong demagnetizing effect resulting from the thin-film sample geometry. ${ }^{19}$ On entering the volume of the ribbon, we observe an increasing diamagnetic response as $h_{\mathrm{ac}}(t)$ (a)

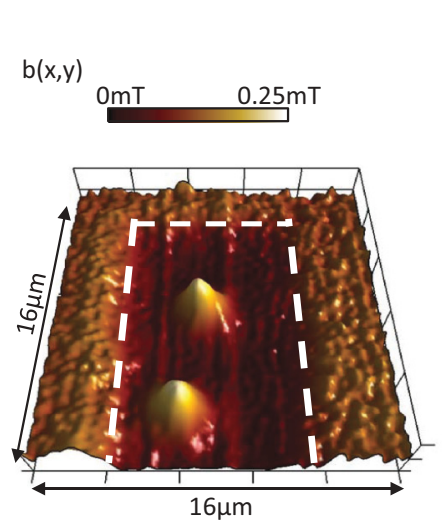

(b)

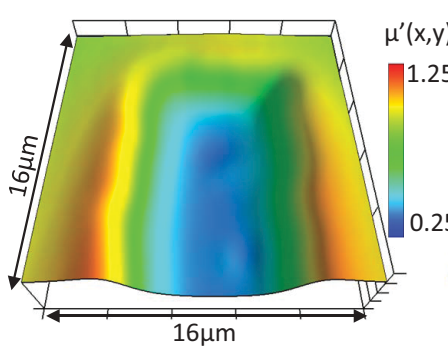

$\mathrm{T}=6.9 \mathrm{~K}$
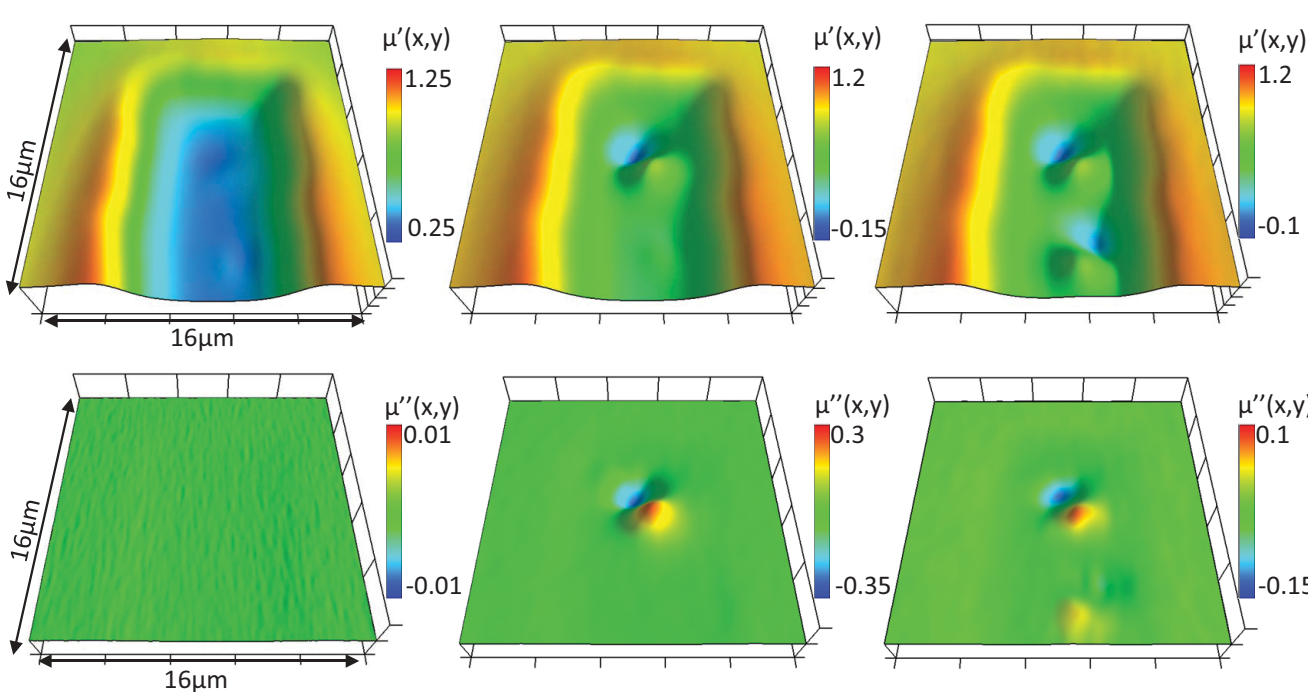

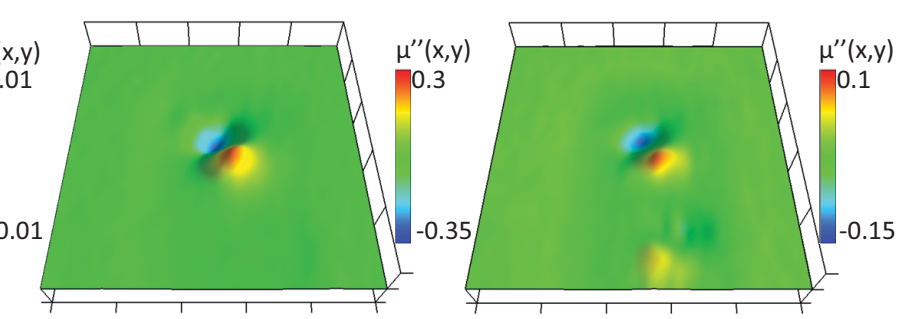

FIG. 1. (Color online) (a) Scanning Hall probe microscopy image of the local induction, $b_{z}(x, y)$, acquired during shaking with an external applied ac field of amplitude, $h_{\mathrm{ac}}=0.1 \mathrm{mT}$, and with frequency, $f=77.123 \mathrm{~Hz}$ at a temperature of $T=6.7 \mathrm{~K}$. The initial vortex distribution is obtained by performing a field cool in an external applied dc magnetic field, $H=0.13 \mathrm{mT}$. The white dashed line indicates the border of the $\mathrm{Pb}$ ribbon. (b) Simultaneously acquired maps of the real part of the relative permeability, $\mu_{r}^{\prime}$ (top row) and the imaginary part of the relative permeability, $\mu_{r}^{\prime \prime}$ (bottom row), for different temperatures (left to right): $T=6.7 \mathrm{~K}, 6.9 \mathrm{~K}$, and $7.0 \mathrm{~K}$. 
gets shielded by the screening currents. At the center of the $\mathrm{Pb}$ ribbon, a maximum diamagnetic response due to the screening current of $\mu_{r}^{\prime}(x, y)=0.27$ at $T=6.7 \mathrm{~K}$ is reached, indicating an incomplete field expulsion. An important observation in Fig. 1(b) is that the shielding currents do not show any contributing signal in $\mu_{r}^{\prime \prime}(x, y)$ for all temperatures, indicating that they are, within our experimental resolution, perfectly in-phase with the ac excitation and as such are nondissipative.

Within the ribbon volume the induced screening currents, $\mathbf{j}(t)$, will periodically shake the vortices, with a force $\mathbf{f}_{\mathbf{L}}(t)=$ $\mathbf{j}(t) \times \boldsymbol{\phi}_{0}$. The ac dynamics of the vortices will crucially depend on the thermodynamical parameters of the SC system and the properties of the drive. For the described experimental parameters, the fingerprint of their motion in the SSM images consists of two distinct unidirectional spots of opposite polarity surrounding the equilibrium vortex position. The inductive response can be easily interpreted. An area with a response exceeding the ac response of the screening currents, $\mu_{r}^{\prime}(x, y)>$ $\mu_{r}^{\prime}(x, y)_{s}$, corresponds with a vortex, carrying an intrinsic positive local induction, moving in-phase with $h_{\mathrm{ac}}(t)$ within this area. A region with a response lower than the ac response of the screening currents, $\mu_{r}^{\prime}(x, y)<\mu_{r}^{\prime}(x, y)_{s}$, in some cases resulting even in a local negative permeability, $\mu_{r}^{\prime}(x, y)<0$, indicates that $b_{z}(x, y, t)$ increases (decreases) on decreasing (increasing) instantaneous $h_{\mathrm{ac}}(t)$, corresponding with a vortex moving in antiphase with $h_{\mathrm{ac}}(t)$ within this area. A similar unique local negative $\mu_{r}^{\prime}(x, y)$ response, but on a substantially larger spatial scale, has already been observed in the ac dynamics of flux droplets in the presence of a geometrical barrier. ${ }^{17}$ From thermodynamical considerations an overall integrated response between 0 and 1 is expected for $\left\langle\mu_{r}^{\prime}\right\rangle$. Note, however, that the meaning of the complex permeability as a macroscopic thermodynamical variable is lost in this local limit. On integrating the local signal over the whole scan area the expected non-negative response for $\left\langle\mu_{r}^{\prime}\right\rangle$ and $\left\langle\mu_{r}^{\prime \prime}\right\rangle$ is recovered. This connection between $\left\langle\mu_{r}\right\rangle$ as the integrand of the "local" permeability, $\mu_{r}(x, y)$, which is directly related to the microscopic vortex dynamics, is used in theoretical models to explain the fingerprints of different dynamical VL regimes in measurements of the global ac susceptibility and can be studied now directly by SSM. In sharp contrast to the screening currents' response, the vortices do leave a fingerprint in $\mu_{r}^{\prime \prime}(x, y)$ for sufficiently high temperatures. As such, the harmonic approximation of their motion exhibits an out-of-phase component. This indicates that vortex motion is accompanied by a dissipative process. The irreversible response disappears below $T<6.8 \mathrm{~K}$, here the ac response of the vortices is weak and, within the experimental resolution, perfectly in phase. The particular depth and shape of the local pinning potential each vortex experiences has a profound effect on the ac dynamics, i.e., at $T=6.9 \mathrm{~K}$ only one of the two vortices present in our scan area is shaken by $h_{\mathrm{ac}}$. The nature of the microscopic processes resulting in vortex dissipation, each having a characteristic time, is a longstanding discussion of interest. ${ }^{20-22}$

In general, vortex dynamics can be described by a phenomenological force balance equation of the form ${ }^{23}$ :

$$
m \ddot{\mathbf{x}}(t)-\mathbf{f}_{\mathbf{L}}(t)+\nabla U(j=0)=-\eta \dot{\mathbf{x}}(t)+\boldsymbol{\xi}(t),
$$

where $\mathbf{x}(t)$ is the vortex position. Here the left-hand side describes the conservative part of the dynamics, including a combination of an inertial term, the periodic Lorentz drive, $\mathbf{f}_{\mathbf{L}}(t)$, and the local potential, $U(j=0)$, the vortex experiences due to a combination of interactions with other vortices, the surface, and the local quenched disorder. For small excitations the local potential in the equation of motion can be approximated by a harmonic potential with spring constant $\alpha_{L}$, called the Labusch constant. ${ }^{24}$ The inertial term, including the mass per unit length of a vortex, $m$, is pure effective in nature, as a vortex is unable to sustain its existence outside the condensate medium. It is accepted to be very small ${ }^{25,26}$ so there is a short initial period of acceleration needed to reach the steady-state motion we consider. The right-hand side of Eq. (2) describes the effects of the nonconservative environment presenting energy dissipation and modeled as a combination of a viscous friction, $\eta$, and a random thermal force, $\boldsymbol{\xi}(t) .{ }^{27}$ It is this right-hand side of the equation of motion which describes the coupling to the environment. The linear approximation to the steady-state solution of Eq. (2) has the following general form ${ }^{11,28}$ :

$$
\mathbf{x}(\mathbf{t})=-\left(\frac{\alpha_{L}}{1-i / \omega \tau_{1}}+i \omega \eta\right)^{-1} \mathbf{f}_{\mathbf{L}}(t) .
$$

Here $\tau_{1}=\left(\eta / \alpha_{L}\right) I_{0}^{2}\left[U(j) / 2 k_{B} T\right]$ is a characteristic relaxation time related to thermally activated hopping of the vortex, $I_{0}(x)$ is the modified Bessel function, and $U(j)$ describes an effective activation energy which is a combination of the intrinsic pinning potential energy, $U(j=0)$, and the Lorentz force energy resulting from the induced super currents, $\mathbf{j}(t)$.

The solution, given by Eq. (3), directly shows the outof-phase component in the linear response induced by the aforementioned dissipative mechanisms. The term $i \omega \eta$ represents the viscous damping mechanism. It is connected by an elementary model developed by Bardeen and Stephen ${ }^{21}$ with resistive processes in the normal core and by a more rigorous analysis with a finite intrinsic relaxation time of the SC order parameter. $^{22}$ This dissipative process has a typical short characteristic time of the order of, $\tau_{p}=\eta / \alpha_{L} \leqslant 0.1 \mu \mathrm{s} .{ }^{23,29}$ For the applied low driving frequency, $f=77.123 \mathrm{~Hz}$, the restoring force dominates over the viscous drag force, as $\omega \ll 1 / \tau_{p}$, and this term can be neglected. The term $i / \omega \tau_{1}$ is related to thermally activated vortex hopping across an effective activation barrier, following the classic ideas of Anderson and $\mathrm{Kim}^{30}$ and results from $\boldsymbol{\xi}(t)$ in Eq. (2). This activated dissipation process is typically associated with longer characteristic time scales. ${ }^{31}$ Under certain conditions it is expected to contribute substantially in our low-frequency SSM experiment.

At low temperatures, when $U(j) \gg k_{B} T$ and thermally activated flux motion can be neglected, $\tau_{1}$ diverges exponentially and the character of the ac response, $\mathbf{x}(\mathbf{t})=-\alpha_{L} \mathbf{f}_{\mathbf{L}}(t)$, is a pure reversible harmonic motion, as described by Campbell and Evetts. ${ }^{10}$ This behavior explains the absence of a response in the SSM images of $\mu_{r}^{\prime \prime}(x, y)$ for $T<6.8 \mathrm{~K}$, while a response is still visible in $\mu_{r}^{\prime}(x, y)$. As the temperature rises, the thermal activation energy decreases and $1 / \omega \tau_{1}$ becomes appreciable, meaning thermally activated vortex jumps between metastable states come into play and contribute substantially to the dissipation process. This explains the observed out-ofphase component for $T>6.8 \mathrm{~K}$. Figure 2 shows a close-up of 

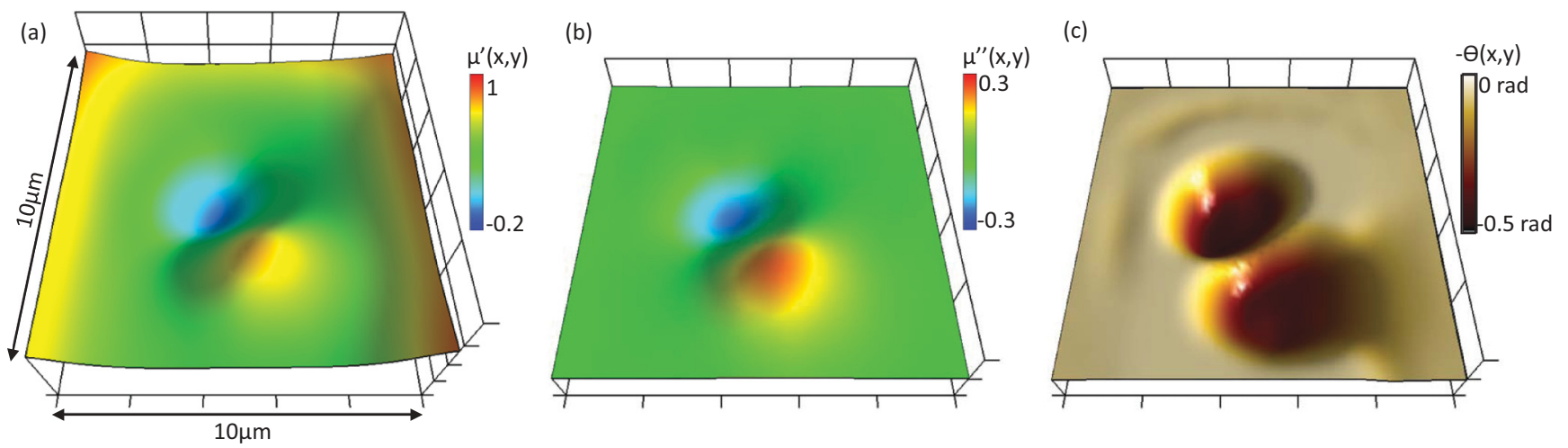

FIG. 2. (Color online) (a) Scanning susceptibility microscopy image of the real part of the relative permeability, $\mu_{r}^{\prime}$, for a single vortex on shaking with an external ac magnetic field of amplitude $h_{\mathrm{ac}}=0.1 \mathrm{mT}$ and frequency $f=77.123 \mathrm{~Hz}$ at a temperature of $T=6.9 \mathrm{~K}$. The initial vortex distribution is obtained by performing a field cool in an external applied dc magnetic field, $H=0.13$ mT. (b) Simultaneously acquired map of the imaginary part of the relative permeability, $\mu_{r}^{\prime \prime}$. (c) Calculated spatial dependence of minus the phase angle.

the ac response of a single vortex for $T=6.9 \mathrm{~K}$ and the corresponding spatial dependence of the calculated phase, where we use a cutoff for $\left|\mu_{r}^{\prime}(x, y)\right|<0.15$ to limit the divergence of arctan and we subtracted the contribution of the screening currents in $\mu_{r}^{\prime}(x, y)$. As shown in Fig. 2(c), the obtained phase shift is $\Theta=-0.5 \mathrm{rad}$. From Eq. (2), the phase shift between the response and the drive is given by $\Theta=-\arctan \left(1 / \tau_{1} \omega\right)$. As $\tau_{p} \leqslant 0.1 \mu \mathrm{s}$, we obtain a lower limit for the effective activation barrier height of $U(j) \geqslant 8.50 \times 10^{-3} \mathrm{eV} \sim$ $14.3 k_{B} \mathrm{~T}$, similar to typical average effective barrier heights found in the literature by macroscopic measurements. ${ }^{32}$

The temperature dependence of the phase shift shows a maximum at $T=6.85 \mathrm{~K}$. Optimal energy dissipation is expected when the driving frequency matches the characteristic frequency of our vortex system, it is, when the resonant absorption condition, $\omega \tau_{1}=1$, is fulfilled. As the driving frequency is fixed, we approach or detune from the resonant absorption condition by changing $\tau_{1}$ with temperature. The nonmonotonic temperature dependence of the phase shift reflects the nontrivial temperature dependencies of the different factors contributing in $\tau_{1}$.

In summary, we explored the microscopic linear response of a SC ribbon to an external applied alternating magnetic field on decreasing temperature. A clear dichotomy between the ac response of the vortices and the screening currents was observed using the phase-sensitive character of the SSM technique. The observed out-of-phase response of the vortices, which was absent for the reversible screening currents, directly shows the local dissipation of the vortex motion. As viscous losses can only account for a small fraction of the observed dissipation at the used experimental excitation frequency, the observed large phase lag, $\Theta=-0.5 \mathrm{rad}$, is explained by thermally activated vortex hopping over pinning centers. As such, a new and powerful tool is introduced and employed, allowing us to investigate for the first time vortex dynamics with single-vortex resolution. In addition, this opens new and exciting possibilities to study locally loss mechanisms in a variety of interesting magnetic systems (e.g., magnetic domain walls). ${ }^{33,34}$

\section{ACKNOWLEDGMENTS}

We acknowledge the Methusalem funding by the Flemish government and the Flemish Science Foundation, FWO-Vl, for financial support. C.C.d.S.S. acknowledges support from the Brazilian science agencies $\mathrm{CNPq}$ and FACEPE. J.V.d.V and J.G. acknowledge support from FWO-Vl.
${ }^{1}$ Richard P. Feynman, Robert B. Leighton, and Matthew Sands, The Feynman Lectures on Physics, Vol. 1 (Addison-Wesley, Boston, 1966), chap. 44.

${ }^{2} \mathrm{M}$. Tinkham, Introduciton to Superconductivity (Dover, New York, 2004).

${ }^{3}$ B. D. Josephson, Phys. Lett. 16, 242 (1965).

${ }^{4}$ M. Fox, Optical Properties of Solids (Oxford University Press, Oxford, UK, 2001), chap. 1.

${ }^{5}$ Herbert B. Callen and Theodore A. Welton, Phys. Rev. 83, 34 (1951).

${ }^{6}$ F. Gömöry, Supercond. Sci. Technol. 10, 523 (1997).

${ }^{7}$ M. Marchevsky, M. J. Higgins, and S. Bhattacharya, Nat. Phys. 409, 591 (2001).

${ }^{8}$ A. E. Koshelev and V. M. Vinokur, Phys. Rev. Lett. 73, 3580 (1994).
${ }^{9}$ G. Pasquini, L. Civale, H. Lanza, and G. Nieva, Phys. Rev. B 59, 9627 (1999).

${ }^{10}$ A. M. Campbell and J. E. Evetts, Adv. Phys. 21, 199 (1972).

${ }^{11}$ C. J. van der Beek, V. B. Geshkenbein, and V. M. Vinokur, Phys. Rev. B 48, 3393 (1993).

${ }^{12}$ E. H. Brandt, Phys. Rev. Lett. 67, 2219 (1991).

${ }^{13}$ J. R. Kirtley, Rep. Prog. Phys. 73, 126501 (2010).

${ }^{14}$ M. P. DeFeo and M. Marchevsky, Phys. Rev. B 73, 184409 (2006).

${ }^{15}$ R. B. G. Kramer, G. W. Ataklti, V. V. Moshchalkov, and A. V. Silhanek, Phys. Rev. B 81, 144508 (2010).

${ }^{16}$ R. B. G. Kramer, A. V. Silhanek, W. Gillijns, and V. V. Moshchalkov, Phys. Rev. X 1, 021004 (2011).

${ }^{17}$ N. Morozov, E. Zeldov, D. Majer, and B. Khaykovich, Phys. Rev. Lett. 76, 138 (1996). 
${ }^{18}$ M. Marchevsky, P. H. Kes, and J. Aarts, Physica C 282-287, 2083 (1997).

${ }^{19}$ E. H. Brandt and M. Indenbom, Phys. Rev. B 48, 12893 (1993).

${ }^{20}$ Y. B. Kim, C. F. Hempstead, and A. R. Strnad, Phys. Rev. 139, A1163 (1965).

${ }^{21}$ J. Bardeen and M. J. Stephen, Phys. Rev. 140, A1197 (1965).

${ }^{22}$ M. Tinkham, Phys. Rev. Lett. 13, 804 (1964).

${ }^{23}$ J. I. Gittleman and B. Rosenblum, Phys. Rev. Lett. 16, 734 (1966).

${ }^{24}$ R. Labusch, Cryst. Lattice Defects 1, 1 (1969).

${ }^{25}$ H. Suhl, Phys. Rev. Lett. 14, 226 (1965).

${ }^{26}$ D. Golubchik, E. Polturak, and G. Koren, Phys. Rev. B 85, 060504(R) (2012).

${ }^{27}$ P. Fulde, L. Pietronero, W. R. Schneider, and S. Strässler, Phys. Rev. Lett. 35, 1776 (1975).
${ }^{28}$ M. W. Coffey and J. R. Clem, Phys. Rev. Lett. 67, 386 (1991).

${ }^{29}$ A. A. Awad, F. G. Aliev, G. W. Ataklti, A. Silhanek, V. V. Moshchalkov, Y. M. Galperin, and V. Vinokur, Phys. Rev. B 84, 224511 (2011).

${ }^{30} \mathrm{P}$. W. Anderson and Y. B. Kim, Rev. Mod. Phys. 36, 39 (1964).

${ }^{31}$ Peter Hänggi, Peter Talkner, and Michal Borkovec, Rev. Mod. Phys. 62, 251 (1990).

${ }^{32}$ M. Lange, M. J. Van Bael, A. V. Silhanek, and V. V. Moshchalkov, Phys. Rev. B 72, 052507 (2005).

${ }^{33}$ W. Kleemann, J. Rhensius, O. Petracic, J. Ferré, J. P. Jamet, and H. Bernas, Phys. Rev. Lett. 99, 097203 (2007).

${ }^{34}$ E. Saitoh, H. Miyajima, T. Yamaoka, and G. Tatara, Nat. Phys. 432, 203 (2004). 